PROCEEDINGS OF THE

AMERICAN MATHEMATICAL SOCIETY

Volume 130, Number 8, Pages 2419-2424

S 0002-9939(02)06327-X

Article electronically published on February 4, 2002

\title{
BIFURCATION SETS OF DEFINABLE FUNCTIONS IN O-MINIMAL STRUCTURES
}

\author{
JESÚS ESCRIBANO \\ (Communicated by Carl G. Jockusch, Jr.)
}

\begin{abstract}
In this work we answer a question stated by Loi and Zaharia concerning trivialization of definable functions off the bifurcation set: we prove that definable functions are trivial off the bifurcation set, and the trivialization can be chosen definable.
\end{abstract}

\section{INTRODUCTION}

Let $U \subset \mathbb{R}^{n}$ be an open subset and $g: U \rightarrow \mathbb{R}$ a $C^{p}$ function. We are interested in the following problem: when is $g$ a trivial fibration (in a neighbourhood of a point)? If $t_{0} \in \mathbb{R}$ is a regular value of $g$ and there exists an open neighbourhood $V \subset \mathbb{R}$ of $t_{0}$ such that the restriction $g: g^{-1}(V) \rightarrow V$ is a $C^{p}$ trivial fibration, we will call $t_{0}$ a $C^{p}$ typical value. Otherwise $t_{0}$ is called a $C^{p}$ atypical value of $g$, and the set of $C^{p}$ atypical values of $g$ is called the $C^{p}$ bifurcation set of $g$. We are interested in identifying the bifurcation sets. We study this problem in an o-minimal context, that is, for definable functions on an o-minimal expansion of a real closed field.

Let us recall the situation for arbitrary $C^{p}$ functions on $\mathbb{R}$, not necessarily definable. Let $U \subset \mathbb{R}^{n}$ be an open set and fix a $C^{1}$ function $\rho: U \rightarrow \mathbb{R}$ such that

$$
\text { for each } r \in \mathbb{R} \text {, the "ball" } B_{r}^{\rho}=\{u \in U: \rho(u) \leq r\} \text { is compact. }
$$

We denote by $S_{r}^{\rho}=\{x \in U: \rho(x)=r\}$ the corresponding "sphere". For a $C^{1}$ function $g: U \rightarrow \mathbb{R}$, we define

$$
M(g ; \rho)=\{x \in U: \exists \lambda \in \mathbb{R}, \operatorname{grad} g(x)=\lambda \operatorname{grad} \rho(x)\} .
$$

For a sequence $\left\{y^{k}\right\} \subset M(g ; \rho)$ we consider the conditions

$$
\lim _{k \rightarrow \infty} \rho\left(y^{k}\right)=\infty \quad \text { and } \quad \lim _{k \rightarrow \infty} g\left(y^{k}\right)=c,
$$

and define

$$
S_{g ; \rho}=\left\{c \in \mathbb{R}: \text { there exists a sequence }\left\{y^{k}\right\} \subset M(g ; \rho) \text { that verifies (2) }\right\} .
$$

Received by the editors February 2, 2001 and, in revised form, February 28, 2001 and March $12,2001$.

2000 Mathematics Subject Classification. Primary 03C64; Secondary 58C25.

The author was partially supported by DGICYT, PB98-0756-C02-01. 
Let $\Sigma_{g}$ be the set of critical values of $g$. Then we have the following theorem ([10] $1.3])$ :

Theorem 1.1. Let $U \subset \mathbb{R}^{n}$ be an open subset and let $g, \rho: U \rightarrow \mathbb{R}$ be $C^{p+1}$ functions for some $p \in \mathbb{N} \cup\{\infty\}$, where $\rho$ is as above. Let us assume that $\Sigma_{\rho}$ is bounded. Then for any open interval $J \subset g(U) \backslash\left(\Sigma_{g} \cup S_{g ; \rho}\right)$, the restriction

$$
g: g^{-1}(J) \rightarrow J
$$

is a $C^{p}$ trivial fibration.

We recall that a $C^{p}$ function $g: U \rightarrow J$, where $U \subset R^{n}$ and $J \subset R$ are open, is $C^{p}$ trivial if there exists a $C^{p}$ diffeomorphism $\gamma=\left(\gamma_{0}, g\right): U \rightarrow g^{-1}(a) \times J$, with $a \in J$; in other words, we have the following commutative diagram:

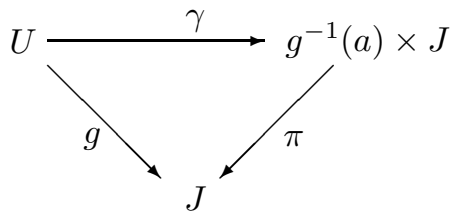

where $\pi$ is the projection $(x, t) \mapsto t$.

We turn now to the definable case. Let $\mathcal{S}$ be an o-minimal structure expanding the real closed field $R([7])$. We consider $\mathcal{D}_{\mathcal{S}}^{p}$ functions (that is, definable and $C^{p}$ functions) defined over $R$ (see [2] and 4 for results on differentiability on real closed fields); we simply write $\mathcal{D}^{p}$ if $\mathcal{S}$ is clear from the context. We prove a definable version of Theorem 1.1] asked for by Loi and Zaharia in [10].

Theorem 1.2. Let $U \subset R^{n}$ be an open definable subset and let $g, \rho: U \rightarrow R$ be $\mathcal{D}^{p}$ functions for some $p>0$, where $\rho$ verifies condition (1). Then for any open interval $J \subset g(U) \backslash\left(\Sigma_{g} \cup S_{g ; \rho}\right)$, the restriction

$$
g: g^{-1}(J) \rightarrow J
$$

is $\mathcal{D}^{p}$ trivial.

Of course, $\mathcal{D}^{p}$ trivial means as above, plus all data definable.

We wish to thank Professors Michel Coste and Jesús M. Ruiz for their help and support during the preparation of this paper.

\section{BASIC RESULTS}

A classical tool used to study triviality problems is integration of vector fields ([1]). However, we cannot use this tool in the o-minimal context. Following the ideas from [5], we substitute integration of vector fields by the definable spectrum and the construction of definable models.

The construction of the definable spectrum is done in [3, following the better known one for the semialgebraic case (2]). We can embed $R^{m}$ in a compactification $\widetilde{R^{m}}$ whose points $\alpha$ are ultrafilters of definable sets. Given a definable subset $A \subset R^{m}$, we can consider the set $\widetilde{A}=\left\{\alpha \in \widetilde{R^{m}}: \alpha \ni A\right\} \subset \widetilde{R^{m}}$.

Take $\alpha \in \widetilde{R^{m}}$. Two definable functions $f: A \rightarrow R, g: B \rightarrow R$, for $A, B \in \alpha$, are equivalent if there exists a definable subset $C \in \alpha, C \subset A \cap B$, such that $f_{\mid C}=g_{\mid C}$. The set $k(\alpha)$ of equivalence classes for this equivalence relation is a real closed field ([4 Th. 5.8]). We denote by $f(\alpha)$ the equivalence class of $f: A \rightarrow R$. 
If $f=\left(f_{1}, \ldots, f_{n}\right): A \rightarrow R^{n}$ is a definable mapping, $A \in \alpha$, we denote by $f(\alpha)$ the point $\left(f_{1}(\alpha), \ldots, f_{n}(\alpha)\right) \in k(\alpha)^{n}$.

A definable family of definable subsets of $R^{n}$, parametrized by $R^{m}$, is a definable subset $X \subset R^{m} \times R^{n}$. The fiber of $X$ at a point $t \in R^{m}$ is the definable subset $X_{t}=\left\{x \in R^{n}:(t, x) \in X\right\} \subset R^{m}$. The fiber of $X$ at $\alpha \in \widetilde{R^{m}}$ is the set $X_{\alpha}$ of those $f(\alpha) \in k(\alpha)^{n}$ such that there exists $A \in \alpha$ on which $f$ is defined and $(t, f(t)) \in X$ for all $t \in A$. Let $\mathcal{S}_{n}(\alpha)$ be the collection of all fibers $X_{\alpha}$, for $X$ a definable subset of $R^{m} \times R^{n}$. Then $\left(\mathcal{S}_{n}(\alpha)\right)_{n \in \mathbb{N}}$ is an o-minimal structure expanding the real closed field $k(\alpha)$ (4 , Th. 5.8]).

Given a definable function $f: U \subset R^{n} \rightarrow R^{m}$, we can see $f$ as a definable family of definable subsets $\left\{U_{t}\right\}_{t \in R^{m}}$, where $U_{t}=f^{-1}(t)$. For such a definable family, we can define the fiber of the family at a point of $\widehat{R^{m}}$. The philosophy behind the use of definable spectrum is the following: given a definable family of definable sets, parametrized by $R^{m}$, and given a point $\alpha \in \widetilde{R^{m}}$, the fiber of the family at $\alpha$ verifies a given property (expressed by first-order formulas) if and only if there exists a definable subset $S \subset R^{m}, S \in \alpha$, such that all the fibers at points $t \in S$ verify the same property. Furthermore, from the study of the properties of the generic fibers, we can obtain not just fiberwise properties, but global ones. An example of this is the following:

Proposition 2.1. Let $B \subset R^{m}$ and $X \subset B \times R^{n}$ be definable subsets. Let $\alpha \in \widetilde{B}$ be such that $X_{\alpha}$ is a $\mathcal{D}^{p}$ submanifold of $k(\alpha)^{n}$. Then there exists a $\mathcal{D}^{p}$ submanifold $M \subset R^{m}, M \subset B$, such that $M \in \alpha, X \cap\left(M \times R^{n}\right)$ is a $\mathcal{D}^{p}$ submanifold of $M \times R^{n}$ and the projection $\pi: X \cap\left(M \times R^{n}\right) \rightarrow M$ is a submersion.

Proof. See [8, Prop. 5.1.6]. Compare with [5, Prop. 2.2].

The construction of definable models means the following. Let $\left(R^{\prime}, \mathcal{S}^{\prime}\right)$ and $(R, \mathcal{S})$ be two o-minimal structures expanding real closed fields such that $R^{\prime} \subset R$. We will say that $(R, \mathcal{S})$ is an elementary extension of $\left(R^{\prime}, \mathcal{S}^{\prime}\right),\left(R^{\prime}, \mathcal{S}^{\prime}\right) \prec(R, \mathcal{S})$, if, for each $n$, there exists an extension mapping $\mathcal{S}_{n}^{\prime} \rightarrow \mathcal{S}_{n}: A^{\prime} \mapsto A_{R}^{\prime}$ such that:

1. $A_{R}^{\prime} \cap R^{\prime n}=A^{\prime}$ for each $A^{\prime} \in \mathcal{S}_{n}^{\prime}$,

2. $A^{\prime} \mapsto A_{R}^{\prime}$ commutes with boolean operations,

3. $\left(A^{\prime} \times B^{\prime}\right)_{R}=A_{R}^{\prime} \times B_{R}^{\prime}$ for each $A^{\prime} \in \mathcal{S}_{n}^{\prime}, B^{\prime} \in \mathcal{S}_{m}^{\prime}$,

4. if $\pi^{\prime}: R^{\prime n+1} \rightarrow R^{\prime n}$ and $\pi: R^{n+1} \rightarrow R^{n}$ are the projections on the first $n$ coordinates, and $A^{\prime} \in \mathcal{S}_{n+1}^{\prime}$, then $\pi\left(A_{R}^{\prime}\right)=\pi^{\prime}\left(A^{\prime}\right)_{R}$,

5. if $A^{\prime}$ is semialgebraic, $A_{R}^{\prime}$ is the usual extension of semialgebraic sets ([2]),

6. for each $B \in \mathcal{S}_{m}$, there exists $n \in \mathbb{N}, A^{\prime} \in \mathcal{S}_{m+n}^{\prime}$ and $a \in R^{n}$ such that $B=\left\{x \in R^{m}:(x, a) \in A_{R}^{\prime}\right\}$.

We remark that, if $A^{\prime} \in \mathcal{S}_{n}^{\prime}$ is empty, its projection to ${R^{\prime}}^{0}=R^{0}$ (singleton) is empty, and hence, by (4), $A^{\prime}{ }_{R}$ is also empty.

In [5] the authors construct "Nash models" of Nash manifolds over smaller real closed ground fields. In our o-minimal context, we have the next result (see [8, Th. 5.3.2]; compare with [5, Th. 3.1]).

Theorem 2.2. Let $\left(R^{\prime}, \mathcal{S}^{\prime}\right) \prec(R, \mathcal{S})$ be an elementary extension. Let $X \subset R^{n}$ be a $\mathcal{D}_{\mathcal{S}}^{p}$ submanifold. Then there exists a $\mathcal{D}_{\mathcal{S}^{\prime}}^{p}$ submanifold $Y$ such that $Y_{R}$ is $\mathcal{D}_{\mathcal{S}}^{p}$ diffeomorphic to $X$. 
There exists a strong relation between construction of definable models and definable triviality of definable families, as we can see in the following

Theorem 2.3 ([8, Th. 5.1.10]; compare with [5, Th. 2.4]). Take $k \in \mathbb{N}$ fixed. The following properties are equivalent:

(i) Let $(R, \mathcal{S})$ be an o-minimal structure expanding the real closed field $R, p, n \in$ $\mathbb{N}$. Let $B \subset R^{p}$ and $X \subset B \times R^{n}$ be definable subsets such that for each $b \in B$, $X_{b}$ is a $\mathcal{D}^{r}$ manifold of dimension $k$. Then there exists a finite stratification $B=\bigcup_{i \in I} M_{i}$ of $B$ in $\mathcal{D}^{r}$ manifolds such that each $X \cap\left(M_{i} \times R^{n}\right)$ is a $\mathcal{D}^{r}$ manifold and there is a $\mathcal{D}^{r}$ trivialization

$$
h_{i}: X \cap\left(M_{i} \times R^{n}\right) \rightarrow F_{i} \times M_{i}
$$

for some $\mathcal{D}^{r}$ manifold $F_{i}$.

(ii) For any elementary extension $\left(R^{\prime}, \mathcal{S}^{\prime}\right) \prec(R, \mathcal{S})$, each $\mathcal{D}_{\mathcal{S}}^{r}$ manifold $V \subset R^{n}$ of dimension $k$ is $\mathcal{D}_{\mathcal{S}}^{r}$ diffeomorphic to the extension to $(R, \mathcal{S})$ of a $\mathcal{D}_{\mathcal{S}^{\prime}}^{r}$ manifold $V^{\prime} \subset R^{\prime m}$.

The above result, plus the Approximation Theorem for o-minimal structures (9]), allows us to prove the following triviality result:

Theorem 2.4 ([9, Th. 2.1]). Let $p: M \rightarrow R^{l}$ be a surjective proper $\mathcal{D}^{r}$ submersion. Then $p$ is $\mathcal{D}^{r}$ trivial.

\section{Proof of Theorem 1.2}

In [10, Th. 1.5] the authors prove that the sets $\Sigma_{g}$ and $S_{g ; \rho}$ are finite. Let $J$ be an open interval as above. For each $t \in J, U_{t}=g^{-1}(t)$ is a $\mathcal{D}^{p}$ manifold. Hence, for each $\alpha \in \widetilde{J}, U_{\alpha}$ is a $\mathcal{D}^{p}$ manifold. By Theorem 2.2 there exists a $\mathcal{D}^{p}$ manifold $F$ and a $\mathcal{D}^{p}$ diffeomorphism $\gamma: F_{k(\alpha)} \rightarrow U_{\alpha}$. The $\mathcal{D}^{p}$ manifold $F_{k(\alpha)}$ is the generic fiber of the trivial family $J \times F$ at the point $\alpha$. Thus, the definable families $\left\{U_{t}\right\}_{t \in J}$ and $J \times F$ are $\mathcal{D}^{p}$ diffeomorphic at the generic point $\alpha$, and, by the standard use of the definable spectrum (see for instance [8, Th. 5.1.10]), both families are diffeomorphic on some definable subset, that is, there exists a definable subset $I^{\alpha} \subset R, I^{\alpha} \in \alpha$, and a $\mathcal{D}^{p}$ diffeomorphism $h: F \times I^{\alpha} \rightarrow g^{-1}\left(I^{\alpha}\right)$. By o-minimality, we can assume that the sets $I^{\alpha}$ are singletons or open intervals. Hence, by the compactness of the definable spectrum, we obtain that $g$ is $\mathcal{D}^{p}$ trivial outside a finite number of values $\left\{a_{1}, \ldots, a_{k}\right\}$.

Consider a value $a=a_{i}$. As $a \notin S_{g, \rho}$, we can find $\varepsilon>0$ and $K>0$ big enough such that

$$
(g, \rho): g^{-1}(a-\varepsilon, a+\varepsilon) \cap \rho^{-1}(K,+\infty) \longrightarrow(a-\varepsilon, a+\varepsilon) \times(K,+\infty)
$$

is a proper definable submersion. Hence it is $\mathcal{D}^{p}$ trivial (2.4), that is, there exist a definable $\mathcal{D}^{p}$ diffeomorphism

$$
\begin{aligned}
& \Theta=(\Delta, g, \rho): g^{-1}(a-\varepsilon, a+\varepsilon) \cap \rho^{-1}(K,+\infty) \\
& \longrightarrow\left(g^{-1}(a) \cap \rho^{-1}(K+1)\right) \times(a-\varepsilon, a+\varepsilon) \times(K,+\infty) .
\end{aligned}
$$

From $\Theta$ we obtain a $\mathcal{D}^{p}$ diffeomorphism

$$
\Theta_{0}=(\Delta, \rho)_{\mid g^{-1}(a)}: g^{-1}(a) \cap \rho^{-1}(K,+\infty) \longrightarrow\left(g^{-1}(a) \cap \rho^{-1}(K+1)\right) \times(K,+\infty)
$$


and hence we can consider the $\mathcal{D}^{p}$ diffeomorphism

$$
\begin{aligned}
\mu=\left(\mu_{0}, g\right): g^{-1}(a-\varepsilon, a+\varepsilon) & \cap \rho^{-1}(K,+\infty) \\
& \longrightarrow\left(g^{-1}(a) \cap \rho^{-1}(K,+\infty)\right) \times(a-\varepsilon, a+\varepsilon), \\
x & \longmapsto\left(\Theta_{0}^{-1}(\Delta(x), \rho(x)), g(x)\right) .
\end{aligned}
$$

We can assume that $\mu_{0 \mid g^{-1}(a)}=\mathrm{Id}$.

On the other hand, let us consider the definable manifold with boundary

$$
g^{-1}(a-\varepsilon, a+\varepsilon) \cap \rho^{-1}(-\infty, K+2]
$$

and the definable function

$$
g: g^{-1}(a-\varepsilon, a+\varepsilon) \cap \rho^{-1}(-\infty, K+2] \longrightarrow(a-\varepsilon, a+\varepsilon),
$$

which is a proper submersion and it is also a submersion restricted to the boundary (we again use that $a \notin S_{g, \rho}$; see [1] for basic results on definable manifolds with boundary). Then, by the o-minimal version of [6, Th. 3] (the proof is straightforward), the above restriction is also $\mathcal{D}^{p}$ trivial, that is, there exists a definable diffeomorphism

$$
\begin{aligned}
h= & \left(h_{0}, g\right): g^{-1}(a-\varepsilon, a+\varepsilon) \cap \rho^{-1}(-\infty, K+2] \\
& \longrightarrow\left(g^{-1}(a) \cap \rho^{-1}(-\infty, K+2]\right) \times(a-\varepsilon, a+\varepsilon) .
\end{aligned}
$$

We can assume that $h_{0 \mid g^{-1}(a)}=\mathrm{Id}$ and $\rho \circ h_{0}=\rho$ in an open definable neighbourhood of $\rho^{-1}(K+2)$. In fact, we can assume that $\rho \circ h_{0}=\rho$ on $\rho^{-1}(K, K+2)$. This can be achieved by shrinking the neighborhood of $a$ and multiplying $\rho$ by a constant.

We want to glue these two trivializations in order to obtain a definable trivialization over $(a-\varepsilon, a+\varepsilon)$. Consider the $\mathcal{D}^{p}$ diffeomorphism

$$
\begin{aligned}
\mu \circ h^{-1}:\left(g^{-1}(a) \cap \rho^{-1}(K, K+2)\right) & \times(a-\varepsilon, a+\varepsilon) \\
\longrightarrow & \left(g^{-1}(a) \cap \rho^{-1}(K, K+2)\right) \times(a-\varepsilon, a+\varepsilon), \\
(x, t) & \longmapsto(\tau(x, t), t) .
\end{aligned}
$$

Let $\phi: R \rightarrow R$ be a $\mathcal{D}^{p}$ function, $0 \leq \phi \leq 1$, such that $\phi \equiv 0$ on $\left(-\infty, K+\frac{1}{2}\right]$ and $\phi \equiv 1$ on $\left[K+\frac{3}{2},+\infty\right)$. We then define $\tau^{*}(x, t)=\tau(x, \phi(\rho(x)) t+(1-\phi(\rho(x))) a)$ for $x \in g^{-1}(a) \cap \rho^{-1}(K, K+2)$ and $t \in(a-\varepsilon, a+\varepsilon)$. Take $x \in g^{-1}(a) \cap \rho^{-1}(K, K+2)$ and define $\gamma_{0}(x)=\tau^{*} h(x)$. If $\phi=1, \gamma_{0}(x)=\tau\left(h_{0}(x), g(x)\right)=\mu_{0}(x)$. If $\phi=0$, $\gamma_{0}(x)=\tau\left(h_{0}(x), a\right)$. By definition, $\left(\tau\left(h_{0}(x), a\right), a\right)=\mu \circ h^{-1}\left(h_{0}(x), a\right)=\mu\left(h_{0}(x)\right)=$ $\left(\mu_{0} h_{0}(x), a\right)=\left(h_{0}(x), a\right)$, hence $\tau\left(h_{0}(x), a\right)=h_{0}(x)$.

We can then extend $\gamma_{0}$ to $g^{-1}(a-\varepsilon, a+\varepsilon)$ by

$$
\gamma_{0}(x)= \begin{cases}\mu_{0}(x) & \text { if } \rho(x) \in[K+2,+\infty), \\ \gamma_{0}(x) & \text { if } \rho(x) \in[K, K+2], \\ h_{0}(x) & \text { if } \rho(x) \in(-\infty, K] .\end{cases}
$$

Then $\gamma=\left(\gamma_{0}, g\right)$ is a $\mathcal{D}^{p}$ trivialization of $g$ over $(a-\varepsilon, a+\varepsilon)$.

In this way, we can cover $J$ by a finite number of open intervals such that $g$ is $\mathcal{D}^{p}$ trivial over each of them. To finish the proof, we must glue these trivializations. But, in the o-minimal category, we have an approximation theorem of $\mathcal{D}^{p}$ functions by $\mathcal{D}^{p+1}$ functions ([9]), so that we can repeat the argument in [5, Th. 2.4] to obtain a global trivialization. 


\section{REFERENCES}

1. A. Berarducci, M. Otero Intersection theory for o-minimal manifolds, Annals of Pure and Applied Logic 107, num. 1-3 (2001), 87-119. CMP 2001:07

2. J. Bochnak, M. Coste, M.-F. Roy: Real Algebraic Geometry, Ergeb. Math. Grenzgeb. (3) 36, Springer-Verlag, Berlin - Heidelberg - New York, 1998. MR 2000a:14067

3. M. Coste: Topological types of fewnomials, Singularities Symposium - Łojasiewicz 70, Banach Center Pub. 44, 81-92 (1998). MR 2000b:14075

4. M. Coste: An introduction to o-minimal geometry, Dottorato di Ricerca in Matematica, Dip. Mat. Univ. Pisa, Instituti Editoriali e Poligrafici Internazionali (2000).

5. M. Coste, M. Shiota: Nash triviality in families of Nash manifolds, Invent. Math. 108 (1992), 349-368. MR 93e:14066

6. M. Coste, M. Shiota: Thom's first isotopy lemma: a semialgebraic version, with uniform bound, in Real Analytic and Algebraic Geometry (Ed. F. Broglia, M. Galbiati, A. Tognoli), Walter de Gruyter, Berlin, 1995, 83-101. MR 96i:14047

7. L. van den Dries: Tame topology and o-minimal structures, London Math. Soc. Lecture Note 248. Cambridge Univ. Press (1998). MR 99j:03001

8. J. Escribano: Trivialidad definible de familias de aplicaciones definibles en estructuras ominimales, Ph. D. dissertation, Universidad Complutense de Madrid (2000). Also available at http://www.ucm.es/info/dsip/directorio/Personales/jemweb/escriban.html.

9. J. Escribano Martínez: Approximation theorems in o-minimal structures, preprint. Also available at http://www.ucm.es/info/dsip/directorio/Personales/jemweb/escriban.html.

10. T. L. Loi, A. Zaharia: Bifurcation sets of functions definable in o-minimal structures, Illinois Journal of Mathematics, Vol. 42, Num. 3, Fall 1998. MR 99f:58023

11. A. Némethi, A. Zaharia: On the bifurcation set of a polynomial function and Newton boundary, Publ. Res. Inst. Math. Sci. 26, no 4 (1990), 681-689. MR 92c:32046

Departamento de Sistemas Informáticos y Programación, Facultad de CC. MatemátiCas, Universidad Complutense, E-28040 Madrid, Spain

E-mail address: escribano@sip.ucm.es 